\title{
Validação Experimental de um Modelo de Análise de Sinais em Amplificadores Raman
}

\author{
Carlos Eduardo S. Castellani, Shirley P. N. Cani, Maria José Pontes, Maria Thereza M. R. Giraldi, \\ Andrés Pablo L. Barbero e Marcelo E. V. Segatto
}

\begin{abstract}
Resumo-Esse artigo apresenta a validação experimental de um modelo numérico de propagação de sinais em amplificadores Raman. O modelo inclui interações entre bombeios e sinais, além de geração de ruído de emissão espontânea amplificada. A validação do modelo numérico é feita através da comparação do ganho on-off e da relação sinal-ruído óptica calculados, com valores obtidos experimentalmente. Sistemas contendo amplificadores Raman distribuídos com $50 \mathrm{~km}$ e $100 \mathrm{~km}$ de fibra monomodo padrão considerando um sinal de dados, e dois bombeios co-propagantes foram analisados. A avaliação da penalidade do diagrama de olho é efetuada a partir de resultados calculados.
\end{abstract}

Palavras chaves-Amplificador Raman, amplificação óptica, fibra óptica.

\begin{abstract}
This paper presents an experimental validation of a numerical model for the signal propagation in Raman amplifiers. The model includes interactions between pump and signal and the generation of noise from amplified spontaneous emission. The validation of the model has been obtained through the comparison between the on-off gain and the optical signal noise rate calculated and measured. Systems with distributed Raman amplifiers with 50 $\mathbf{k m}$ and $100 \mathrm{~km}$ of single-mode standard fiber, considering one data signal, and two pumps in a co-propagating configuration scheme have been analyzed. The analysis of the eye diagram penalty has been done with the numerical model.
\end{abstract}

Key words-Raman amplification, optical amplification, optical fiber.

\section{INTRODUÇÃO}

$\mathrm{E}$ $\mathrm{m}$ comunicações ópticas sempre houve uma grande ${ }_{\text {preocupação em se atingir distâncias (L) cada vez maiores }}$ com taxas de transmissões (B) de dados também maiores. Nesse sentido, o desenvolvimento de amplificadores ópticos foi

Carlos E. S. Castellani, Shirley P. N. Cani, Maria José Pontes e Marcelo E. V. Segatto, Departamento de Engenharia Elétrica - CTII, Universidade Federal do Espírito Santo - UFES, Campus de Goiabeiras, Vitória, Brasil, E-mails: ceduardosc@terra.com.br, segatto@ele.ufes.br. M.Thereza M.R. Giraldi, Seção de Engenharia Elétrica, Instituto Militar de Engenharia IME, Rio de Janeiro, RJ, Brasil, E-mail: mtmrocco@ime.eb.br. A. Pablo L. Barbero, Departamento de Engenharia de Telecomunicações, Universidade Federal Fluminense - UFF, E-mail: pablo@telecom.uff.br

Trabalho parcialmente financiado pela CAPES e CNPq. extremamente importante, pois permitiu estender significativamente o produto $\mathrm{B} \times \mathrm{L}$. Durante o período de implantação dos primeiros amplificadores ópticos em sistemas comerciais, deu-se preferência à utilização de amplificadores a fibra dopada com Érbio - EDFA (Erbium Doped Fiber Amplifier) [1] ao invés dos amplificadores Raman [2]. Os amplificadores Raman necessitavam de lasers de bombeio operando na região de $1450 \mathrm{~nm}$ com potências muito elevadas, algo que inicialmente não existia. Porém, o avanço da tecnologia desses lasers de bombeamento fez com que o problema fosse contornado, possibilitando assim o aumento da utilização de amplificadores Raman. Com isso vieram muitos benefícios, pois os amplificadores Raman apresentam algumas vantagens em relação aos EDFAs, como uma ampla banda de amplificação, o que é altamente desejável no caso de sinais WDM (Wavelength Division Multiplexing) [1], melhor relação sinal-ruído, freqüência central de amplificação flexível e alta capacidade de transmissão. Isso tudo sem precisar alterar os enlaces de fibra óptica já instalados, pois os amplificadores Raman distribuídos (DRAs - Distributed Raman Amplifiers), por exemplo, utilizam a própria fibra do enlace como meio de amplificação.

Esse artigo apresenta a validação experimental de um modelo numérico de propagação proposto para a análise de sinais em amplificadores Raman. Estão incluídos na análise do desempenho dos sistemas os seguintes efeitos que afetam a propagação de sinais modulados sobre a portadora óptica: interações entre bombeios e sinais, efeitos dispersivos de alta ordem, efeitos não-lineares tais como a auto modulação de fase (SPM - Self-Phase Modulation), modulação cruzada de fase (XPM - Cross-Phase Modulation), espalhamento Raman estimulado (SRS- Stimulated Raman Scattering), e amplificação do ruído de emissão espontânea de Raman. A inclusão deste último efeito no modelo está entre as principais contribuições do trabalho.

A validação do modelo é obtida através da comparação dos resultados obtidos em simulação computacional com dados obtidos experimentalmente. Os parâmetros de projeto do amplificador utilizados na comparação foram a OSNR (Optical Signal to Noise Ratio) e o ganho on-off do sistema.

Adicionalmente, é apresentado um resultado de penalidade 
do sinal propagante, obtido através da solução numérica do modelo apresentado neste trabalho. Embora este resultado não seja comparado a dados experimentais, ele é um indicativo das influências negativas dos efeitos dispersivos e não lineares sobre os sistemas com sinais modulados.

Respeitando as condições experimentais disponíveis em laboratório, foram feitos testes apenas com amplificadores Raman distribuídos em enlaces de fibra monomodo padrão, ou SMF (Single Mode Fiber), contendo um laser de sinal e dois lasers de bombeamento na configuração co-propagante. Tanto o laser de sinal quanto os lasers de bombeio operaram no modo de onda contínua ou CW (continous wave). Outras configurações de montagem dos amplificadores Raman tais como DRAs com bombeio contra-propagante considerando múltiplos canais de entrada e vários lasers de bombeio, ou mesmo amplificadores concentrados, poderão ser exploradas em possíveis trabalhos futuros.

Este trabalho está organizado da seguinte forma: a Seção II apresenta a metodologia utilizada para a obtenção e análise dos resultados; a Seção III apresenta o esquema experimental montado em laboratório, assim como os resultados e as discussões; e por fim, a conclusão é apresentada na Seção IV.

\section{Metodologia}

O modelo numérico apresentado neste trabalho foi desenvolvido tomando como base as equações acopladas de campo conhecidas como equações de Schrödinger, conforme equação (1) [3].

$$
\begin{aligned}
& \frac{\partial A_{v}}{\partial z}-d_{r e f, v} \frac{\partial A_{v}}{\partial T}+\frac{j}{2} \beta_{2_{v}} \frac{\partial^{2} A_{v}}{\partial T^{2}}-\frac{j}{6} \beta_{3_{v}} \frac{\partial^{3} A_{v}}{\partial T^{3}}=-\frac{\alpha_{v}}{2} A_{v} \\
& +j \gamma_{v}\left[\left|A_{v}\right|^{2}+\left(\sum_{v=1}^{N c} 2\left|A_{v}\right|^{2}\right)-2\left|A_{v}\right|^{2}\right] A_{v} \\
& +A_{v} \sum_{\mu>v} \frac{C_{R_{\mu \nu}}}{2 \Gamma}\left|A_{\mu}\right|^{2} \\
& -A_{v} \sum_{\mu<v} \frac{\omega_{v}}{\omega_{\mu}} \frac{C_{R_{v \mu}}}{2 \Gamma}\left|A_{\mu}\right|^{2} \\
& -A_{\nu} \sum_{\mu<\nu} \frac{\omega_{v}}{\omega_{\mu}} \frac{C_{R_{v \mu}}}{2 \Gamma} 4 N_{E_{\mu}}\left[1+\frac{1}{\exp \left[\frac{h(v-\mu)}{k T}\right]-1}\right]
\end{aligned}
$$

As equações de campo (1) governam as amplitudes de canais modulados propagantes em um meio dispersivo e não linear. Neste trabalho, portanto, as equações de campo foram modificadas para simular a propagação dos bombeios e do sinal em amplificadores Raman considerando a geração e a amplificação do ruído de emissão espontânea. É considerado na solução do modelo somente o ruído de emissão espontânea gerado na freqüência do sinal, e a conseqüente influência deste efeito na potência do bombeio.

Embora o termo relacionado à amplificação da emissão espontânea - ASE (Amplified Spontaneous Emission) não esteja inserido na equação (1), este efeito é incluído na solução numérica das equações de amplitude, de acordo com o procedimento descrito a seguir.

Na equação (1) $A_{v}$ é a amplitude complexa, $\gamma_{v}$ é o parâmetro de não linearidade, $\alpha_{v}$ é o coeficiente de atenuação, $\omega_{v}$ é a freqüência angular referente a freqüência linear $v, \omega_{\mu}$ é a freqüência angular referente a freqüência linear $\mu$, e $N_{C}$ é o número total de amplitudes complexas propagando ao longo da fibra (incluindo sinal e bombeio). $C_{R \mu v}=g_{\mu \nu} / A_{\text {eff }}$ é a eficiência do ganho Raman entre as freqüências $\mu$ e $\nu$, e $g_{\mu \nu}$ é o ganho Raman na freqüência $v$ devido ao bombeio na freqüência

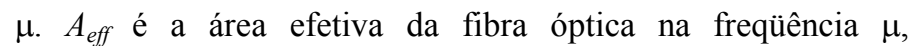
$N_{E v}=h v \Delta v$ é a potência de ruído devido à emissão espontânea (ASE - Amplified Spontaneous Emission) gerada em uma banda $\Delta v$ em torno da freqüência $v ; h$ é a constante de Planck, $k$ é a constante de Boltzmann. $T$ é a temperatura absoluta da fibra, fixada em $300 \mathrm{~K}$ e $\Gamma$ é o fator de polarização, que é assumido ter valor igual a 2 (transmissão despolarizada) [4]. O termo $d_{r e f, v}$ se refere à diferença entre as velocidades de grupo do canal de referência e os demais (walk-off). O canal de referência adotado corresponde ao menor comprimento de onda de bombeio ou a menor frequência utilizada.

Em (1) está incluído: dispersão da velocidade de grupo (GVD - Group Velocity Dispersion), dispersão de alta ordem $\beta_{3 v}$, auto modulação de fase (SPM), modulação cruzada de fase (XPM), espalhamento de Raman estimulado (SRS), e amplificação do ruído de emissão espontânea de Raman.

Para solucionar numericamente a equação (1) utilizou-se o software Matlab e o método iterativo split-step Fourier, que consiste em dividir a fibra em pequenos passos e em cada passo calcular os efeitos de dispersão e não linearidades separadamente.

O termo de amplitude relacionado ao ruído ASE pode ser identificado como $A_{A S E, v}$. Esta amplitude foi modelada como uma variável aleatória complexa com potência média igual a $N_{E v}$. A inserção da contribuição de ruído foi obtida da seguinte forma: a cada passo, obtinha-se a amplitude do sinal transmitido, e da mesma forma a amplitude de ruído equivalente. Ambas as amplitudes complexas foram transformadas em contribuição de potência. Os valores em potência foram somados e a potência resultante foi novamente convertida em amplitude, condicionado a que a fase do sinal resultante permanecesse a mesma obtida antes da inserção do 
ruído. E assim este processo foi repetido sucessivamente nos passos seguintes.

Esta aproximação assume que a fase do sinal não é afetada pela contribuição do ruído. Esta suposição pode ser aceita em sistemas reais, uma vez que a ASE gerada em pequenos passos é muito pequena quando comparada com a amplitude do sinal.

Neste trabalho os dados obtidos pela análise de sinais são comparados a resultados experimentais $\mathrm{CW}$. A validade de realizar a comparação entre um sistema modulado (bits de sinal) e um sistema $\mathrm{CW}$ foi verificada a partir da análise de uma sequência de bits " 1 " e outra de bits " 0 " de modo a simular uma situação CW [5]. Os resultados obtidos foram então comparados com aqueles obtidos através da análise de potência e as potências médias obtidas nas duas situações concordaram perfeitamente. As comparações aqui realizadas levam em consideração tais resultados.

No cálculo numérico do ganho on-off $\left(\mathrm{G}_{\text {on-off }}\right)$ dos sistemas, foi utilizado a equação (2) [2], que mostra justamente a relação em unidades de decibel $(\mathrm{dB})$ entre a potência do sinal na saída com e sem a presença do bombeio.

$$
G_{\text {on-off }}=10 \log _{10} \frac{P_{v}(L)}{P_{v, \text { aten }}(L)}
$$

Onde $P_{v}(L)$ é a potência do sinal obtida no final da fibra após solução numérica da equação (1), e $P_{v, \text { aten }}(L)$ é a potência do sinal atenuado dada pela equação (3),

$$
P_{v, \text { aten }}(L)=P_{v}(0) \times e^{-\alpha_{v} L} .
$$

Nas equações (2) e (3) $L$ é o comprimento total do enlace de fibra, e $P_{v}(0)$ é a potência inicial do sinal na entrada da SMF.

A relação sinal ruído óptico (OSNR) foi calculada a partir da equação (4) [6], que mostra justamente a razão em unidades de decibel $(\mathrm{dB})$ entre a potência do sinal e a potência do ruído na saída do amplificador. A potência de ruído ASE $P_{A S E, V}$ foi calculada numericamente acoplando um sinal com potência igual a zero na entrada e medindo a potência do sinal na saída. Dado que o ruído ASE é gerado pela potência de bombeio e não pela potência de sinal, e que estamos trabalhando com amplificadores Raman fora da região de saturação (não ocorre depleção do bombeio devido à potência do sinal), esta aproximação no cômputo da potência de ASE pode ser adotada. A banda de geração da ASE em torno da freqüência do sinal foi assumida $\Delta v=1 \mathrm{~nm}$.

$$
O S N R=10 \log _{10} \frac{P_{v}(L)}{P_{A S E, v}(L)}
$$

\section{RESULTADOS E DISCUSSÕES}

A Figura 1 mostra a configuração do amplificador Raman distribuído utilizado nos testes em laboratório. O esquema foi montado utilizando um enlace de SMF com dois lasers CW de bombeio co-propagantes centrados, respectivamente, em 1448.5 $\mathrm{nm}$ e $1456.5 \mathrm{~nm}$. Para evitar que o ganho Raman sofresse deterioração devido à influência de polarização, os lasers de bombeio foram combinados em um despolarizador. Um laser CW sintonizável dentro da banda de $1525 \mathrm{~nm}$ a $1565 \mathrm{~nm}$ foi utilizado como sinal. Os lasers de bombeio e de sinais foram acoplados na entrada da fibra através de um acoplador WDM. A potência dos sinais na saída da fibra foi observada em um analisador de espectro óptico - OSA (Optical Spectrum Analyzer).

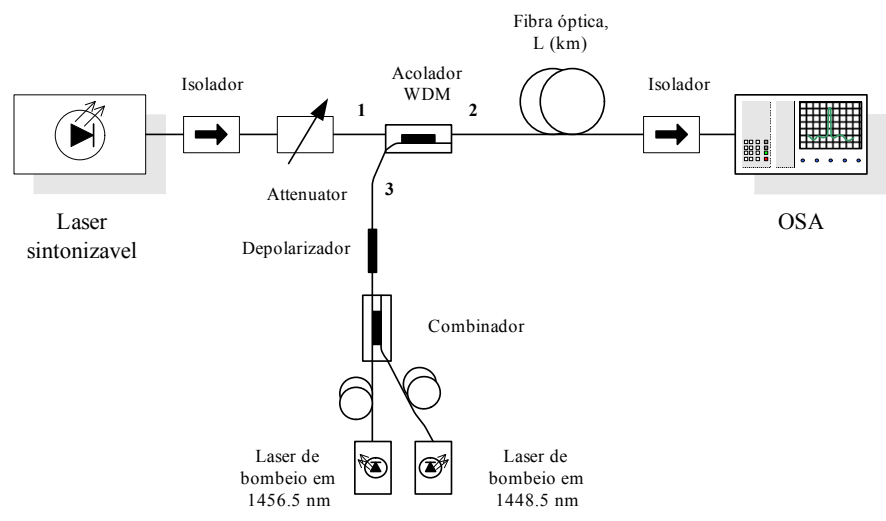

Fig. 1: Esquema montado para a realização dos experimentos.

\section{A. Testes Realizados com $50 \mathrm{~km}$ de SMF}

No primeiro teste experimental, utilizou-se $50 \mathrm{~km}$ de SMF. O laser sintonizável foi centrado em $1552.03 \mathrm{~nm}$ e a potência do sinal foi fixada em $0 \mathrm{dBm}$. A potência dos bombeios foi assumida como sendo a mesma e foi variada entre $20.57 \mathrm{dBm}$ e $24.79 \mathrm{dBm}$. As perdas medidas na fibra foram $0.30 \mathrm{~dB} / \mathrm{km}$ para os comprimentos de onda dos bombeios e $0.23 \mathrm{~dB} / \mathrm{km}$ para os sinais. A eficiência do ganho de Raman $C_{R}=0.4 \mathrm{~W}^{-1} \mathrm{~km}^{-1}$ foi obtida experimentalmente através do procedimento dado por [7].

A solução numérica da equação (1) foi obtida simulando um sinal NRZ (Non Return to Zero) com pulsos super-gaussianos de ordem dois transmitidos a uma taxa de $10 \mathrm{~Gb} / \mathrm{s}$, e dois bombeios $\mathrm{CW}$. Os dados utilizados na simulação foram os mesmos obtidos experimentalmente para $50 \mathrm{~km}$ de fibra.

A Figura 2 mostra a comparação entre o ganho on-off obtido numericamente com o experimental, em função da potência dos 
bombeios. Note que no eixo da abscissa está sendo representada a potência total dos bombeios.

Observa-se na Figura 2 que com o aumento da soma das potências de bombeio, o ganho on-off também aumenta. Isto se deve ao fato de que quanto mais energia é colocada na fibra, mais energia será transmitida para o sinal, fazendo com que este chegue com uma potência maior no final do enlace. E, de acordo com a equação (2), apresentando um ganho on-off mais elevado.

Outro fato importante é que os valores obtidos por simulação, ficaram muito próximos dos valores obtidos experimentalmente, apresentando uma diferença máxima de apenas $0.14 \mathrm{~dB}$.

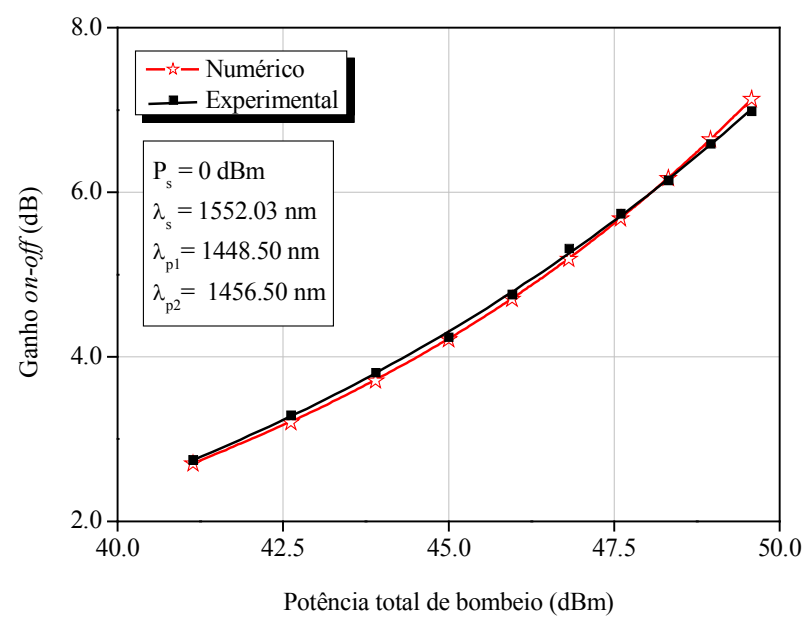

Fig. 2: Ganho on-off simulado e experimental em função da potência total de bombeio para $50 \mathrm{~km}$ de SMF.

\section{B. Testes Realizados com $100 \mathrm{~km}$ de SMF}

Para o segundo teste utilizou-se $100 \mathrm{~km}$ de SMF. Neste experimento variou-se o comprimento de onda dos sinais entre $1530.1 \mathrm{~nm}$ e $1543.3 \mathrm{~nm}$. A potência de cada bombeio foi fixada em $22.5 \mathrm{dBm}$ e a potência dos sinais em $0 \mathrm{dBm}$. Nesse teste as perdas na fibra foram estimadas em $0.26 \mathrm{~dB} / \mathrm{km}$ para os bombeios e $0.23 \mathrm{~dB} / \mathrm{km}$ para os sinais. $\mathrm{O}$ valor de $C_{R}$ foi $\mathrm{o}$ mesmo obtido em $50 \mathrm{~km}$.

Da mesma forma que no teste anterior, a solução numérica da equação (1) foi obtida simulando sinais NRZ (Non Return to Zero) com pulsos super-gaussianos de ordem dois transmitidos a uma taxa de $10 \mathrm{~Gb} / \mathrm{s}$, e dois bombeios $\mathrm{CW}$. Os dados da fibra utilizados na simulação foram os mesmos obtidos experimentalmente para $100 \mathrm{~km}$ de fibra.

A Figura 3 mostra a comparação entre o ganho on-off obtido através de simulação numérica com o experimental, em função dos comprimentos de onda do sinal. É importante ressaltar que os lasers de bombeio foram previamente escolhidos para fornecer ganho plano próximo a $1550 \mathrm{~nm}$ (região de grande interesse em comunicações ópticas), porém os dados experimentais colhidos estavam entre $1530.1 \mathrm{~nm}$ e $1543.3 \mathrm{~nm}$, e por este motivo a Figura 3 não ilustra um ganho plano. Verificase na Figura 3 que também nesse caso o modelo proposto neste trabalho é satisfatório, pois apresenta uma diferença máxima, entre os valores medidos e simulados, de apenas $0.53 \mathrm{~dB}$, o que é uma diferença pequena considerando que estamos comparando resultados numéricos com dados experimentais.

Outro resultado obtido tanto experimentalmente quanto numericamente foi a OSNR em função da variação do comprimento de onda do sinal, cujos resultados estão mostrados na Figura 4.

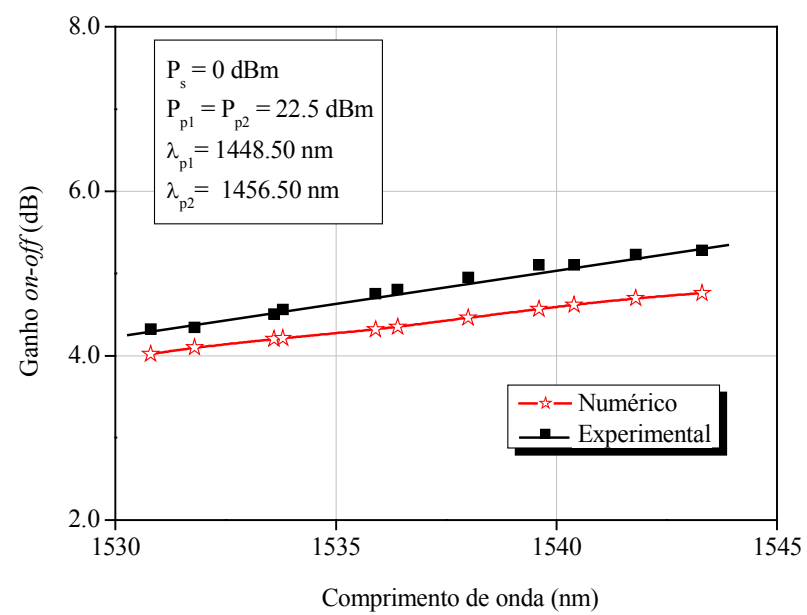

Fig. 3. Ganho on-off simulado e experimental em função do comprimento de onda do sinal para $100 \mathrm{~km}$ de SMF.

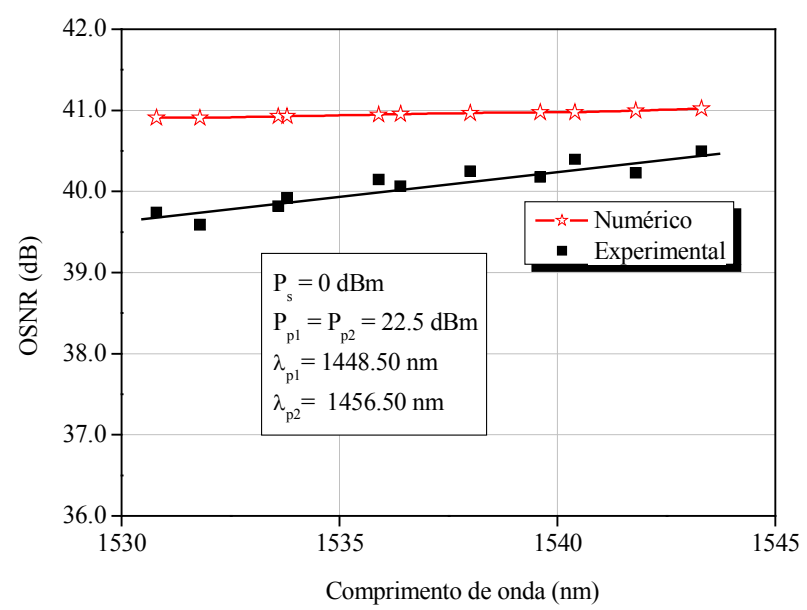

Fig. 4. OSNR numérico e experimental em função do comprimento de onda do sinal para $100 \mathrm{~km}$ de SMF. 
Pode-se observar na Figura 4 que a OSNR cresceu com o aumento do comprimento de onda utilizado. Isto se deve ao fato de, como mostrado na Figura 3, o ganho do amplificador também aumentar nessa região. Apesar da ASE também ser amplificada, a OSNR aumenta indicando que o sinal é amplificado em uma proporção maior. A máxima diferença apresentada entre os resultados numéricos e experimentais na Figura 4 foi de $1,3 \mathrm{~dB}$.

\section{Penalidade de Diagrama de Olho.}

A penalidade de diagrama de olho é uma figura de mérito importante na avaliação do desempenho dos sistemas e é usada como uma forma de se medir quantitativamente a interferência negativa dos efeitos dispersivos e não lineares sobre os sinais em sistemas modulados. A penalidade em $\mathrm{dB}$ indica quanto $\mathrm{o}$ diagrama de olho fechou em relação à sua máxima abertura, que é obtida no início da transmissão dos bits dos sinais. As Figuras 5 (a) e (b) ilustram as penalidades de diagrama de olho em função da soma das potências de bombeio para um sinal em $1552.03 \mathrm{~nm}$ (Fig. 5(a)) e em função do comprimento de onda do sinal quando a potência dos bombeios é fixa em $22.5 \mathrm{dBm}$ por bombeio (Fig. 5(b)). As penalidades foram calculadas ao final de $50 \mathrm{~km} \mathrm{e} 100 \mathrm{~km}$ de SMF.

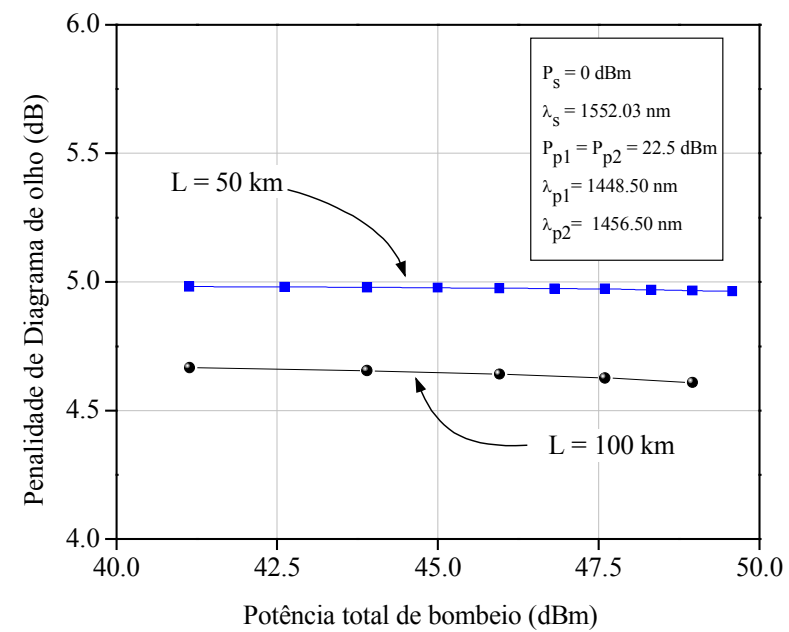

(a)

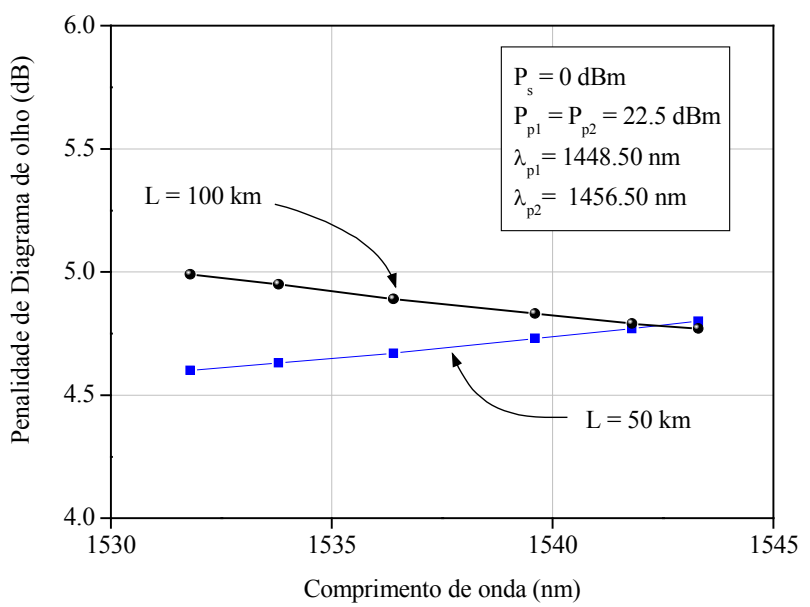

(b)

Fig. 5. Penalidade de diagrama de olho em função (a) da potência dos bombeios e (b) do comprimento de onda.

A comparação da penalidade simulada com dados experimentais não foi possível, uma vez que os lasers utilizados no esquema experimental operaram em regime $\mathrm{CW}$.

Vê-se na Figura 5 que a potência de bombeio não influenciou muito na penalidade de diagrama de olho. Isto acontece porque os bombeios e o sinal estão muito espaçados em freqüência (walk-off com valor alto), fazendo com que o efeito de modulação cruzada de fase seja desprezível [3]. Devido à SMF apresentar alta dispersão no comprimento de onda utilizado, o efeito de mistura de quatro ondas também não influi no resultado de penalidade e, portanto não está sendo considerado em nosso modelo [3]. Portanto os únicos efeitos que tiveram influência na penalidade foram a auto-modulação de fase, que não apresenta forte dependência com a potência de bombeio, e a GVD que não depende da potência dos sinais propagantes.

A penalidade por efeitos não lineares é dependente da potência dos sinais e da proximidade em freqüência entre eles. Em amplificadores Raman com apenas um comprimento de onda de sinal a penalidade será fortemente dependente da potência do sinal, uma vez que a condição de ganho nestes amplificadores é garantir bombeios e sinais afastados em aproximadamente $13 \mathrm{THz}$.

A penalidade por GVD varia com o comprimento de onda de acordo com a curva de dispersão em função do comprimento de onda apresentada em [1]. Em sistemas operando com comprimentos de onda próximos a $1550 \mathrm{~nm}$, a curva de dispersão é aproximadamente plana. Efeitos dispersivos são geralmente eliminados em sistemas ópticos através de técnicas de compensação de dispersão. Em amplificadores Raman distribuídos a dispersão poderá ser compensada utilizando 
algum dispositivo acoplado ao sistema como um módulo compensador de dispersão composto, por exemplo, por uma grade de Bragg e circulador, utilizando compensação eletrônica, ou até mesmo um trecho de fibra compensadora de dispersão, que é a opção mais indicada nos casos de amplificadores Raman, pois pode-se utilizar a fibra compensadora também como meio amplificador.

\section{CONCLUSÕES}

Esse trabalho apresenta um modelo numérico de propagação de sinais em amplificadores Raman. O modelo inclui interações entre bombeios e sinais, efeitos dispersivos e não lineares, além da amplificação do ruído de emissão espontânea na freqüência dos sinais e a interferência deste efeito no comprimento de onda dos bombeios. Foram analisados amplificadores Raman com um sinal de entrada e dois bombeios na configuração copropagante. Os resultados de ganho e OSNR obtidos através do modelo numérico mostraram uma forte concordância com os dados experimentais, apresentando diferenças bem pequenas para diversos parâmetros de entrada do amplificador.

Também foi apresentado um resultado de penalidade obtido através da solução numérica. Este resultado mostra uma estimativa do comportamento dos bits em sistemas com sinais modulados.

A validação completa do modelo de análise de sinais apresentado neste trabalho requer a análise de outras configurações de montagem dos amplificadores Raman tais como DRAs com bombeio contra-propagante considerando múltiplos canais de entrada e vários lasers de bombeio, ou mesmo amplificadores concentrados. Estas análises poderão ser exploradas em possíveis trabalhos futuros.

\section{AGRADECIMENTOS}

Os autores agradecem ao CRT/EMBRATEL por disponibilizar a fibra STD para a realização dos trabalhos experimentais.

\section{REFERÊNCIAS}

[1] G.P. Agrawal, Fiber-Optic Communication Systems. $2^{\text {a. }}$ Ed., New York, John Wiley \& Sons, Inc., 1997.

[2] C. Headley; G. P. Agrawal (editors), Raman Amplification in Fiber Optical Communication Systems. N. Y., Elsevier Academic Press, 2005.

[3] G. P. Agrawal, Nonlinear Fiber Optics, $3^{\text {a. }}$ Ed., San Diego, Academic Press, 2001.

[4] Y. Aoki, "Properties of fiber Raman amplifiers and their applicability to digital optical communication systems. IEEE Journal of Lightwave Technology, v. 6, no. 7, pp. 1225-1239, Jul. 1988.
[5] S. P. N. Cani, M. Freitas, R. T.R. Almeida, and L. C. Calmon, "Raman amplifier performance of dispersion compensating fibers, Proceedings of the 2003 SBMO Microwave and Optoelectronics Conference /IEEE MTT-S International, Vol. 2, pp. 553 - 558, 20-23 Set. Foz do Iguaçu, PR - Brasil, 2003.

[6] S. P. N. Cani, L. C. Calmon, "Analysis of different pumping schemes in distributed Raman amplifiers", Anais do SBMO/IEEE MTT-S International Microwave and Optoelectronics Conference - IMOC'05, Brasília - Brasil, 2005, cd-rom, Jul. 2005.

[7] Y. Kang, Calculation and Measurements of Raman Gain Coefficients of Different Fiber Types, Dissertação de Mestrado em Engenharia Elétrica, Intituto Politécnico da Virginia, Virginia, EUA, Dezembro de 2002. 